\title{
Antimicrobial activity and cytotoxicity of endophytes from Scapania verrucosa Heeg.
}

\author{
J.-G. Wu ${ }^{1 *}$, W. Peng ${ }^{2 *}$, P.-Y. Zeng ${ }^{3}$, Y.-B. $\mathrm{Wu}^{1}, \mathrm{~J} \mathrm{Yi}^{4}$ and J.-Z. Wu ${ }^{1}$ \\ ${ }^{1}$ Academy of Integrative Medicine, \\ Fujian University of Traditional Chinese Medicine, Fuzhou, China \\ ${ }^{2}$ Department of Pharmacology, College of Pharmacy, \\ Third Military Medical University, Chongqing, China \\ ${ }^{3}$ Institute of Traditional Chinese Medicine, \\ Fujian University of Traditional Chinese Medicine, Fuzhou, China \\ ${ }^{4}$ Department of Chemistry and Life Science, Fujian Institute of Education, \\ Fuzhou, China \\ *These authors contributed equally to this study. \\ Corresponding author: J.-Z. Wu \\ E-mail: jinzhongfj@126.com.
}

Genet. Mol. Res. 12 (2): 916-924 (2013)

Received June 26, 2012

Accepted January 5, 2013

Published April 2, 2013

DOI http://dx.doi.org/10.4238/2013.April.2.8

\begin{abstract}
We evaluated the antibacterial activity and cytotoxicity of endophytes isolated from Scapania verrucosa Heeg., which belongs to the liverwort class. A total of 49 endophytic fungi were isolated from S. verrucosa and classified into seven genera and one family in our previous study. In this study, the cytotoxic activity of the endophytes was assessed using the brine shrimp lethality bioassay, seven of which showed potent toxicity against the brine shrimp with 50\% lethal concentration values less than $20 \mu \mathrm{g} / \mathrm{mL}$. T-30 was the most toxic, with a $50 \%$ lethal concentration value of $7.15 \mu \mathrm{g} / \mathrm{mL}$. Moreover, T-27 exhibited the strongest antibacterial activity against Staphylococcus aureus, with minimal inhibitory concentrations below 0.25 and $4 \mathrm{mg} /$ $\mathrm{mL}$, which can inhibit the growth of two standard strains - ATCC 25923 (methicillin-sensitive S. aureus) and ATCC 43300 (methicillin-
\end{abstract}


resistant $S$. aureus) - in a time-dependent manner, respectively. These results suggest that endophytes in $S$. verrucosa are the sources for the production of natural bioactive products and thus warrant further investigation.

Key words: Scapania verrucosa Heeg.; Endophytic fungi; Cytotoxicity; Brine shrimp lethality bioassay; Antibacterial activity;

Staphylococcus aureus

\section{INTRODUCTION}

Endophytic fungi are ubiquitous in plant species, in which they inhabit the tissues beneath the epidermal cell layers without causing any discernible manifestation of disease to the host (Strobel, 2002). Since a paclitaxel (Taxol)-producing endophytic fungal strain was isolated from Taxomyces andreanae (Stierle et al., 1993), a great deal of attention has been focused on endophytic fungi, which can produce active constituents similar to those produced by the host (Yin and Sun, 2011) or other functional metabolites with potential antitumor, antiinflammation, antioxidation, antimicrobial, antiviral, and antipesticide properties (Aly et al., 2011; Wang et al., 2011). Notably, the endophytes from unique habitats in special environments have attracted the most attention (Tan and Zou, 2001).

Scapania verrucosa, which belongs to the liverwort class, grows on forest ground, rocks, and decaying wood and is mainly distributed in south central China, Nepal, and the Himalayan region of Jammu and Kashmir (Gao and Cao, 2000; Söderström et al., 2007). Various rare and novel natural products contained in liverworts display many interesting biological activities such as antimicrobial, cytotoxic, insect antifeedant, muscle relaxing, enzyme inhibitory, apoptosis-inducing, lipoxygenase, calmodulin, hyaluronidase, cyclooxygenase, and thrombin inhibitory properties, and neuritic sprouting. In a previous study, we examined the constituents and antitumor and antifungal activities of the ether extracts of $S$. verrucosa and its endophytic fungus Chaetomium fusiforme. Although the ether extracts of $S$. verrucosa and $C$. fusiforme showed little correlation in chemical composition, both exhibited potent bioactivity, and the antitumor activity of the fungi was even better than that of its host plant (Guo et al., 2008). Furthermore, the ethyl acetate extracts (EEs) of some endophytes dramatically exhibit antioxidant activity (Zeng et al., 2011). To further explore the biological effects of the endophytes from $S$. verrucosa, we evaluated their antibacterial activity against methicillin resistant/ sensitive Staphylococcus aureus (MRSA and MSSA, respectively) as well as their cytotoxicity using the brine shrimp lethality bioassay.

\section{MATERIAL AND METHODS}

\section{Fermentation and extraction}

In our previous study, we isolated endophytic fungi from S. verrucosa and identified it morphologically and molecularly (Zeng et al., 2011). The strains were cultured in potato dextrose broth for 7-14 days at $28^{\circ} \pm 1^{\circ} \mathrm{C}$ with gentle shaking at $1.5 \mathrm{~g}$. The fermentation broth of each strain was centrifuged at $2280 \mathrm{~g}$ for $10 \mathrm{~min}$, and the supernatant obtained was extracted 
three times with ethyl acetate $(\mathrm{v} / \mathrm{v}, 1: 1)$ followed by concentration under reduced pressure to yield the final extract. In this study, the EEs of all isolated endophytic fungi were evaluated for their cytotoxicity by using the brine shrimp lethality bioassay and measurements of antibacterial activity against $S$. aureus.

\section{Brine shrimp lethality bioassay}

Brine shrimp (Artemia salina) eggs were obtained from Qingdao Haidabaichuan Biological Engineering Co., Ltd., Qingdao, Shandong, China. A modified brine shrimp bioassay was performed (Solis et al., 1993). Briefly, EEs were dissolved in dimethylsulfoxide (DMSO; Sigma, USA) and diluted serially $(10,50,100,200$, and $400 \mu \mathrm{g} / \mathrm{mL})$ in artificial seawater. Ten to 15 newly hatched ( $48 \mathrm{~h}$ old) brine shrimp larvae were added to each well and treated with $100 \mu \mathrm{L}$ EE of various concentrations for $24 \mathrm{~h}$ at room temperature. Control brine shrimp were exposed only to $1 \%$ $\mathrm{DMSO} /$ seawater. Under an inverted microscope, the number of inanimate larvae was recorded 24 $\mathrm{h}$ after exposure to the fungal extracts. One hundred microliters of methanol was then added to each well, and after $15 \mathrm{~min}$, the total number of shrimp in each well was counted.

\section{Minimal inhibitory concentration (MIC) test}

The antibacterial activities of the samples were determined using a number of staphylococcal strains, including two standard (ATCC 25923, ATCC 43300) and 10 isolated clinical (SA1-10) strains provided by the Municipal Center for Disease Control and Prevention (Fuzhou, China). MIC determination was carried out using a broth dilution method described previously (Peng et al., 2011). EE dilutions were prepared as follows: 4, 2, 1, 0.5, 0.25, 0.125, and $0.0625 \mathrm{mg} / \mathrm{mL}$. Oxacillin (OXA) was prepared as follows: 1024, 512, 256, 128, 64, 32, $16,8,4,2,1$, and $0.5 \mu \mathrm{g} / \mathrm{mL}$. The sterile broth used for sample dilution was supplemented with DMSO to enhance solubility. MIC values were recorded as the lowest concentration that prevented visible bacterial growth after $24 \mathrm{~h}$ of incubation at $37^{\circ} \mathrm{C}$.

\section{Dynamic bacterial growth assay}

According to the MIC results, the EEs of T-27 and positive control OXA (concentrations of T-27 and OXA used in assays were lower than their MICs) were added to the bacterial suspension (approximately $1.0 \times 10^{6} \mathrm{CFU} / \mathrm{mL}$ ) and cultivated aerobically at $37^{\circ} \mathrm{C}$ in a slowly shaking rocking bed for $24 \mathrm{~h}$. Growth rate was determined by measuring optical density at 600 $\mathrm{nm}\left(\mathrm{OD}_{600}\right)$ at regular intervals (Jiang et al., 2011).

\section{Data analysis}

The $50 \%$ lethal concentration $\left(\mathrm{LC}_{50}\right)$ value at $95 \%$ confidence interval was calculated for each fungi extract with a probit analysis. All experiments were repeated three times.

\section{RESULTS}

Endophytes reportedly have various biological activities. This study was con- 
ducted to evaluate the cytotoxicity and antibacterial activities of the endophytes isolated from $S$. verrucosa. As shown in Table 1, the cytotoxicity was determined using the brine shrimp lethality bioassay. Seven strains - i.e., T-2, T-5, T-21, T-22, T-26, T-30, and T-38 - belonging to Hypocrea rufa, H. viridescens, Tolypocladium sp, Xylaria sp (T-22 and T-26), Chaetomium sp, and Creosphaeria sp were more cytotoxic than the other tested strains, with $\mathrm{LC}_{50}$ values less than $20 \mu \mathrm{g} / \mathrm{mL}$. T-30, belonging to Chaetomium sp, was the most cytotoxic, with an $\mathrm{LC}_{50}$ value of $7.15 \mu \mathrm{g} / \mathrm{mL}$, suggesting that $\mathrm{T}-30$ could produce potent cytotoxic components.

Table1. Brine shrimp lethality bioassay of endophytic fungi from Scapania verrucosa.

\begin{tabular}{|c|c|c|c|c|c|c|c|}
\hline Strain & Taxa & $\mathrm{LC}_{50}(\mu \mathrm{g} / \mathrm{mL})$ & $95 \% \mathrm{CI}$ & Strain & Taxa & $\mathrm{LC}_{50}(\mu \mathrm{g} / \mathrm{mL})$ & $95 \% \mathrm{CI}$ \\
\hline $\mathrm{T}-1$ & Hypocrea viridescens & 308.98 & $259.48-387.69$ & $\mathrm{~T}-26$ & Xylaria $\mathrm{sp} 1$ & 16.67 & $12.83-20.93$ \\
\hline $\mathrm{T}-2$ & Hypocrea rufa & 12.65 & $10.55-32.52$ & $\mathrm{~T}-27$ & Xylaria $\mathrm{sp} 1$ & 24.20 & $19.19-30.81$ \\
\hline $\mathrm{T}-3$ & Xylaria $\mathrm{sp} 1$ & 26.82 & $21.61-32.76$ & $\mathrm{~T}-28$ & Xylariaceae sp 2 & 20.06 & $16.28-24.96$ \\
\hline $\mathrm{T}-4$ & Penicillum sp 1 & 97.14 & $34.11-440.41$ & $\mathrm{~T}-29$ & Nemania diffusa & 65.38 & $56.85-75.06$ \\
\hline $\mathrm{T}-5$ & Hypocrea viridescens & 16.35 & $12.72-27.30$ & $\mathrm{~T}-30$ & Chaetomium sp 1 & 7.15 & $3.58-15.02$ \\
\hline T-6 & Xylariaceae sp 1 & 23.18 & $18.38-28.83$ & $\mathrm{~T}-31$ & Creosphaeria sp & 217.76 & $95.45-383.12$ \\
\hline $\mathrm{T}-7$ & Xylariaceae sp 1 & 41.49 & $38.23-50.78$ & $\mathrm{~T}-32$ & Chaetomium sp 1 & 41.43 & $38.73-46.58$ \\
\hline $\mathrm{T}-8$ & Xylariaceae sp 2 & 53.56 & $48.30-61.30$ & $\mathrm{~T}-33$ & Xylaria sp 1 & 22.15 & $16.12-29.08$ \\
\hline T-9 & Xylariaceae sp 1 & 21.67 & $16.65-27.63$ & $\mathrm{~T}-34$ & Penicillum sp 2 & 42.78 & $36.58-49.52$ \\
\hline $\mathrm{T}-10$ & Xylaria sp 1 & 69.21 & $21.63-147.52$ & $\mathrm{~T}-35$ & Xylariaceae sp 1 & 581.39 & $219.21-988.19$ \\
\hline $\mathrm{T}-11$ & Chaetomium $\mathrm{sp}$ & 270.91 & 224.29-356.59 & $\mathrm{T}-36$ & Xylariaceae sp 1 & 33.38 & $27.51-39.81$ \\
\hline $\mathrm{T}-12$ & Xylariaceae sp 1 & 150.59 & $127.79-180.41$ & $\mathrm{~T}-37$ & Chaetomiun fusiforme & 237.32 & $128.27-328.34$ \\
\hline $\mathrm{T}-13$ & Xylaria sp 3 & 95.06 & $69.43-135.50$ & $\mathrm{~T}-38$ & Creosphaeria sp & 12.03 & $10.75-15.44$ \\
\hline $\mathrm{T}-14$ & Nemania diffusa & 213.06 & $176.52-268.53$ & $\mathrm{~T}-39$ & Creosphaeria sp & 189.60 & $156.83-233.15$ \\
\hline $\mathrm{T}-15$ & Nemania diffusa & 51.11 & $43.91-59.62$ & $\mathrm{~T}-40$ & Creosphaeria sp & 36.55 & $33.12-40.96$ \\
\hline $\mathrm{T}-16$ & Nemania diffusa & 71.87 & $63.13-83.68$ & $\mathrm{~T}-41$ & Chaetomium sp 2 & 242.56 & $107.11-407.67$ \\
\hline $\mathrm{T}-17$ & Xylaria $\mathrm{sp} 1$ & 33.46 & $30.43-36.76$ & $\mathrm{~T}-42$ & Chaetomium sp 2 & 72.45 & $58.72-104.84$ \\
\hline $\mathrm{T}-18$ & Creosphaeria sassafras & 40.10 & $23.16-45.47$ & $\mathrm{~T}-43$ & Creosphaeria sassafras & 69.46 & $59.63-80.42$ \\
\hline T-19 & Tolypoclaidium $\mathrm{sp}$ & 21.11 & $18.00-24.48$ & $\mathrm{~T}-44$ & Chaetomium globosum & 120.67 & $105.44-142.03$ \\
\hline $\mathrm{T}-20$ & Nemania sp & 58.83 & $50.98-65.74$ & $\mathrm{~T}-45$ & Chaetomium globosum & 104.86 & $90.86-121.56$ \\
\hline $\mathrm{T}-21$ & Tolypoclaidium $\mathrm{sp}$ & 16.09 & $10.78-21.68$ & $\mathrm{~T}-46$ & Creosphaeria sassafras & 51.39 & $44.14-59.76$ \\
\hline $\mathrm{T}-22$ & Xylaria sp 1 & 10.48 & $7.09-15.47$ & $\mathrm{~T}-47$ & Nemania $\mathrm{sp}$ & 359.34 & $301.45-463.98$ \\
\hline $\mathrm{T}-23$ & Chaetomium sp 1 & 163.70 & $80.53-350.97$ & $\mathrm{~T}-48$ & Penicillum sp 3 & 622.69 & $287.39-806.52$ \\
\hline $\mathrm{T}-24$ & Chaetomium globosum & 49.41 & $40.85-55.65$ & $\mathrm{~T}-49$ & Creosphaeria sassafras & 57.34 & $50.55-64.60$ \\
\hline $\mathrm{T}-25$ & Creosphaeria sassafras & 35.41 & $5.39-102.95$ & & & & \\
\hline
\end{tabular}

Antibacterial activity was tested against some $S$. aureus strains. According to the results in Table 2, the endophytes displayed moderate antibacterial activity against $S$. aureus, including two standard strains (ATCC 25923 and ATCC 43300) and 10 clinical isolated strains (SA1-10) that were divided into two types (MSSA and MRSA). T-27, belonging to Xylaria sp, displayed the strongest antibacterial activity among these endophytes, inhibiting 8 MSSA and 4 MRSA strains with MICs less than 0.25 and $4 \mathrm{mg} / \mathrm{mL}$, respectively. Moreover, the bacterial dynamic growth curves of ATCC 25923 and ATCC 43300 were observed. The doses of T-27 were $31.25 \mu \mathrm{g} / \mathrm{mL}$ and $1 \mathrm{mg} / \mathrm{mL}$, and the doses of OXA were 0.125 and $128 \mu \mathrm{g} / \mathrm{mL}$ for ATCC 25923 and ATCC 43300, respectively, which were both less than their MICs. As shown in Figures 1 and 2, T-27 and OXA dosedependently decreased the growth rate in ATCC 25923 and ATCC 43300, ATCC 25923 was much more sensitive to T-27 and OXA than ATCC 43300 was. These results indicated that T-27 might be a source for antibacterial drugs. 
J.-G. Wu et al.

Table 2. Results of the minimal inhibitory concentration (MIC).

\begin{tabular}{|c|c|c|c|c|c|c|c|c|c|c|c|c|}
\hline \multirow[t]{3}{*}{ Strains } & \multicolumn{11}{|c|}{$\mathrm{MIC}(\mathrm{mg} / \mathrm{mL})$} & \multirow{3}{*}{ SA- $10 * *$} \\
\hline & \multicolumn{2}{|c|}{$\mathrm{ATCC}^{*} \mathrm{ATCC}^{* *}$} & \multirow{2}{*}{ SA- ${ }^{*}$} & \multirow{2}{*}{ SA-2** } & \multirow{2}{*}{ SA-3* } & \multirow{2}{*}{ SA-4* } & \multirow{2}{*}{ SA-5* } & \multirow{2}{*}{ SA-6* } & \multirow{2}{*}{ SA-7* } & \multirow[t]{2}{*}{$\mathrm{SA}-8^{* *}$} & \multirow[t]{2}{*}{ SA-9* } & \\
\hline & 25923 & 43300 & & & & & & & & & & \\
\hline $\mathrm{T}-1$ & 1 & $>4$ & 0.5 & $>4$ & 2 & 4 & 1 & $>4$ & 2 & $>4$ & $>4$ & $>4$ \\
\hline $\mathrm{T}-2$ & 1 & $>4$ & 2 & $>4$ & 0.5 & $>4$ & 1 & $>4$ & 1 & $>4$ & $>4$ & $>4$ \\
\hline $\mathrm{T}-3$ & 2 & $>4$ & $>4$ & $>4$ & $>4$ & 1 & 2 & $>4$ & $>4$ & $>4$ & $>4$ & $>4$ \\
\hline $\mathrm{T}-4$ & 0.5 & $>4$ & 2 & $>4$ & 4 & 1 & 0.5 & 4 & 2 & $>4$ & 0.25 & $>4$ \\
\hline $\mathrm{T}-5$ & 0.25 & $>4$ & 0.25 & $>4$ & $>4$ & 0.5 & 2 & $>4$ & 1 & $>4$ & $>4$ & $>4$ \\
\hline T-6 & 0.5 & $>4$ & 0.25 & $>4$ & 0.5 & 1 & 1 & $>4$ & 0.5 & $>4$ & 2 & $>4$ \\
\hline $\mathrm{T}-7$ & 1 & $>4$ & 0.25 & $>4$ & 2 & 2 & 2 & $>4$ & 2 & $>4$ & $>4$ & $>4$ \\
\hline $\mathrm{T}-8$ & $>4$ & $>4$ & $>4$ & $>4$ & $>4$ & $>4$ & $>4$ & $>4$ & $>4$ & $>4$ & $>4$ & $>4$ \\
\hline T-9 & 0.5 & $>4$ & 1 & $>4$ & 0.5 & 2 & 2 & 2 & 2 & $>4$ & 1 & $>4$ \\
\hline $\mathrm{T}-10$ & 0.125 & $>4$ & 0.125 & $>4$ & 0.125 & 0.5 & 0.5 & 0.25 & 0.25 & $>4$ & 0.5 & $>4$ \\
\hline T-11 & 1 & $>4$ & 1 & $>4$ & 2 & 1 & 2 & $>4$ & 2 & $>4$ & 2 & $>4$ \\
\hline T-12 & $>4$ & $>4$ & $>4$ & $>4$ & $>4$ & $>4$ & $>4$ & $>4$ & $>4$ & $>4$ & $>4$ & $>4$ \\
\hline T-13 & 1 & $>4$ & 0.5 & $>4$ & 2 & 1 & 2 & 4 & 1 & $>4$ & 1 & $>4$ \\
\hline T-14 & 1 & $>4$ & 0.5 & $>4$ & 1 & 1 & 1 & 2 & 1 & $>4$ & 1 & $>4$ \\
\hline T-15 & 0.125 & $>4$ & 0.125 & $>4$ & 0.125 & 0.5 & 0.25 & 0.5 & 0.5 & $>4$ & 0.25 & $>4$ \\
\hline T-16 & 0.5 & $>4$ & 0.25 & $>4$ & 1 & 0.5 & 1 & 2 & 0.25 & $>4$ & 0.5 & $>4$ \\
\hline T-17 & 0.25 & $>4$ & 0.125 & $>4$ & 0.5 & 0.5 & 0.5 & 1 & 0.5 & $>4$ & 1 & $>4$ \\
\hline T-18 & 1 & $>4$ & 1 & $>4$ & 2 & 0.5 & 2 & 4 & 4 & $>4$ & 4 & $>4$ \\
\hline T-19 & 2 & $>4$ & 2 & $>4$ & 4 & 4 & 4 & $>4$ & 4 & $>4$ & 2 & $>4$ \\
\hline $\mathrm{T}-20$ & 2 & $>4$ & 2 & $>4$ & 2 & 4 & 4 & $>4$ & 4 & $>4$ & 4 & $>4$ \\
\hline $\mathrm{T}-21$ & 4 & $>4$ & 4 & $>4$ & 2 & 4 & 1 & $>4$ & 4 & $>4$ & $>4$ & $>4$ \\
\hline T-22 & $>4$ & $>4$ & $>4$ & $>4$ & $>4$ & $>4$ & $>4$ & $>4$ & $>4$ & $>4$ & $>4$ & $>4$ \\
\hline T-23 & $>4$ & $>4$ & $>4$ & $>4$ & $>4$ & $>4$ & $>4$ & $>4$ & $>4$ & $>4$ & $>4$ & $>4$ \\
\hline T-24 & $>4$ & $>4$ & $>4$ & $>4$ & $>4$ & $>4$ & $>4$ & $>4$ & $>4$ & $>4$ & $>4$ & $>4$ \\
\hline T-25 & $>4$ & $>4$ & $>4$ & $>4$ & $>4$ & $>4$ & $>4$ & $>4$ & $>4$ & $>4$ & $>4$ & $>4$ \\
\hline T-26 & 1 & $>4$ & 1 & $>4$ & 1 & 2 & 1 & 4 & 0.5 & $>4$ & 1 & $>4$ \\
\hline T-27 & 0.125 & 4 & 0.125 & 2 & 0.125 & 0.125 & 0.125 & 0.25 & 0.125 & 4 & 0.125 & 2 \\
\hline T-28 & $>4$ & $>4$ & $>4$ & $>4$ & $>4$ & $>4$ & $>4$ & $>4$ & $>4$ & $>4$ & $>4$ & $>4$ \\
\hline T-29 & 0.25 & $>4$ & 0.5 & $>4$ & 1 & 1 & 1 & 2 & 0.5 & $>4$ & 0.5 & $>4$ \\
\hline T-30 & $>4$ & $>4$ & $>4$ & $>4$ & $>4$ & $>4$ & $>4$ & $>4$ & $>4$ & $>4$ & $>4$ & $>4$ \\
\hline T-31 & $>4$ & $>4$ & $>4$ & $>4$ & $>4$ & $>4$ & $>4$ & $>4$ & $>4$ & $>4$ & $>4$ & $>4$ \\
\hline T-32 & 0.5 & $>4$ & 1 & $>4$ & 2 & 0.5 & 2 & $>4$ & 1 & $>4$ & 4 & $>4$ \\
\hline T-33 & $>4$ & $>4$ & $>4$ & $>4$ & $>4$ & $>4$ & $>4$ & $>4$ & $>4$ & $>4$ & $>4$ & $>4$ \\
\hline T-34 & 1 & $>4$ & 1 & $>4$ & 4 & 2 & 2 & 1 & 2 & $>4$ & 2 & $>4$ \\
\hline T-35 & 2 & $>4$ & 1 & $>4$ & 4 & 1 & 2 & 2 & 2 & $>4$ & 4 & $>4$ \\
\hline T-36 & $>4$ & $>4$ & $>4$ & $>4$ & $>4$ & $>4$ & $>4$ & $>4$ & $>4$ & $>4$ & $>4$ & $>4$ \\
\hline T-37 & $>4$ & $>4$ & $>4$ & $>4$ & $>4$ & $>4$ & $>4$ & $>4$ & $>4$ & $>4$ & $>4$ & $>4$ \\
\hline T-38 & 1 & $>4$ & 4 & $>4$ & 4 & 2 & 4 & $>4$ & $>4$ & $>4$ & $>4$ & $>4$ \\
\hline T-39 & 1 & $>4$ & 1 & $>4$ & 2 & 1 & $>4$ & $>4$ & 0.5 & $>4$ & 4 & $>4$ \\
\hline T-40 & 1 & $>4$ & 1 & $>4$ & 2 & 1 & 4 & $>4$ & 2 & $>4$ & 2 & $>4$ \\
\hline T-41 & $>4$ & $>4$ & $>4$ & $>4$ & $>4$ & $>4$ & $>4$ & $>4$ & $>4$ & $>4$ & $>4$ & $>4$ \\
\hline T-42 & 2 & $>4$ & 1 & $>4$ & 0.5 & 1 & 2 & 0.5 & 0.5 & $>4$ & 1 & $>4$ \\
\hline T-43 & 2 & $>4$ & 2 & $>4$ & $>4$ & 1 & $>4$ & 2 & $>4$ & $>4$ & 2 & $>4$ \\
\hline T-44 & 4 & $>4$ & 2 & $>4$ & $>4$ & $>4$ & 4 & $>4$ & 0.5 & $>4$ & 0.5 & $>4$ \\
\hline T-45 & 0.5 & $>4$ & 0.5 & $>4$ & 1 & 0.5 & 1 & 2 & 0.5 & $>4$ & 1 & $>4$ \\
\hline T-46 & 1 & $>4$ & 0.5 & $>4$ & 1 & 1 & 2 & 4 & 2 & $>4$ & 1 & $>4$ \\
\hline T-47 & 1 & $>4$ & 1 & $>4$ & 0.5 & 1 & 1 & 4 & 1 & $>4$ & 1 & $>4$ \\
\hline T-48 & 2 & $>4$ & 1 & $>4$ & 1 & 1 & 2 & 4 & 1 & $>4$ & 1 & $>4$ \\
\hline T-49 & 2 & $>4$ & 4 & $>4$ & 4 & 2 & 4 & 4 & 2 & $>4$ & 2 & $>4$ \\
\hline OXA & 0.5 & 512 & $<0.5$ & 64 & 1 & $<0.5$ & 1 & 2 & $<0.5$ & 128 & 1 & 256 \\
\hline
\end{tabular}

*MSSA = methicillin-sensitive Staphylococcus aureus; $* * \mathrm{MRSA}=$ methicillin-resistant $S$. aureus; OXA = oxacillin $(\mu \mathrm{g} / \mathrm{mL})$ was used as the positive drug. The concentration of the bacterial suspension was $1.0 \times 10^{5} \mathrm{CFU} / \mathrm{mL}$. 


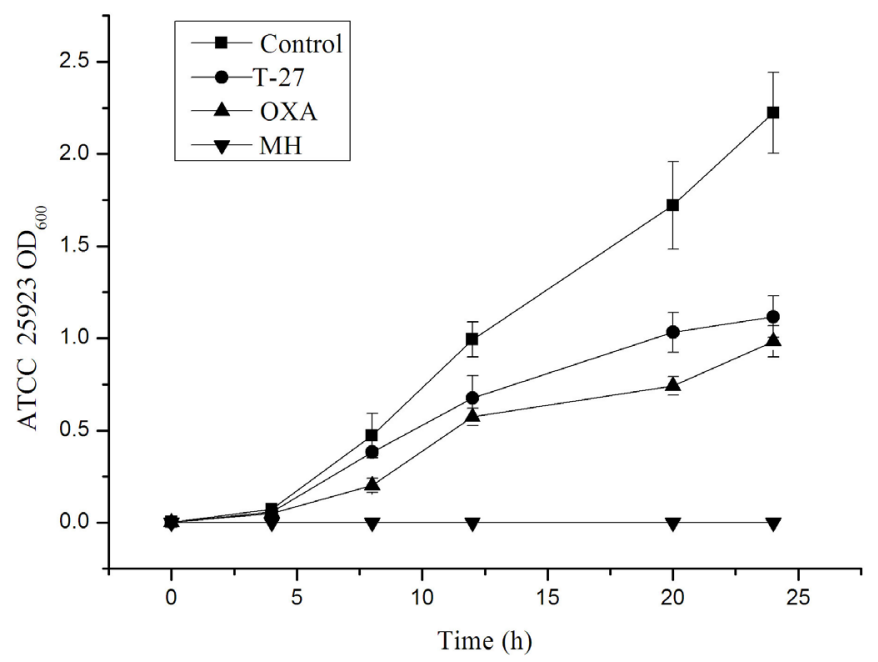

Figure 1. Inhibitory effect of T-27 on the growth of ATCC 25923.

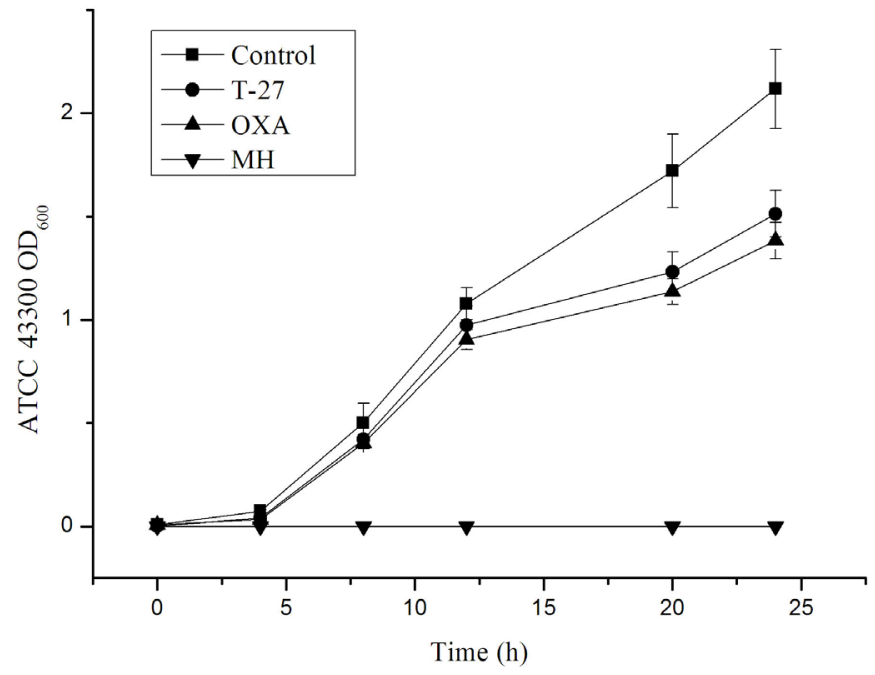

Figure 2. Inhibitory effect of T-27 on the growth of ATCC 43300.

\section{DISCUSSION}

S. verrucosa lives in a special environment, spreading over wild areas and occurring in small populations. Owing to the difficulty of obtaining large amounts of sample, the investigation of chemical composition was halted (Guo et al., 2008). Seeking a way out of the stalemate, researchers have recently recognized endophytic fungi from medical plants as alternative producers of bioactive components (Weber et al., 2007). These fungi can be cultured quickly from 
biomass that can be accumulated through large-scale fermentation (Xu et al., 2009).

Our previous report detailed the separation of 49 endophytic fungi from fresh $S$. verrucosa plants, which were classified into 7 genera (namely, Hypocrea, Penicillium, Tolypocladium, Chaetomium, Xylaria, Nemania, and Creosphaeria) and 1 family (Xylariaceae) using morphological and molecular identification. The majority of these isolated endophytic fungi belonged to the Chaetomium (18.37\%), Creosphaeria (18.37\%), Xylaria (16.33\%), and Xylariaceae $(16.33 \%)$ (Zeng et al., 2011). As a part of our ongoing search for biologically active metabolites, we have reported the antioxidant, antitumor, and antifungal activities of these endophytes. This study was conducted to elucidate the bioactivity more comprehensively. First, for cytotoxicity assessment, the brine shrimp lethality bioassay was selected because it is rapid, simple, easily mastered, inexpensive, and requires only a small amounts of test material. In addition, the bioassay has a robust correlation with cytotoxic activity in some human solid tumors (Ghisalberti, 1993; McLaughlin et al., 1998). Since its introduction, this in vivo lethality test has been successively used to provide a frontline screen that can be supported by more specific and sophisticated bioassays once active compounds have been isolated. As shown in Table 1, 7 strains displayed remarkable cytotoxicity against brine shrimp - particularly, T-30, with an $\mathrm{LC}_{50}$ value of $7.15 \mu \mathrm{g} / \mathrm{mL}$. In our previous study, the Pyricularia oryzae P-2b model and 3-(4,5-dimethylthiazol-2-yl)-2,5-diphenyltetrazolium bromide method were used for in vitro screening of antitumor endophyte strains from $S$. verrucosa [such as T-11, T-12, T-24, T-28, T-31, T-32, T-35, and T-36 (Guo et al., 2009)], revealing the active strains from the brine shrimp lethality bioassay. T-11 was the most active strain and was identified as Chaetomium $\mathrm{sp}$, the same fungal taxon as that of T30, thus suggesting that Chaetomium sp isolated from $S$. verrucosa produces toxic substances that accounted for the significant cytotoxicity of their EEs. Endophytes belonging to Chaetomium sp, isolated from Ginkgo biloba, can produce active constituents with significant cytotoxic activity against brine shrimp (Qin et al., 2009). Accordingly, various bioassays should be performed to understand the biological activities of these compounds more thoroughly. Both T-11 and T-30, belonging to Chaetomium sp, presumably produced the active compounds responsible for the potent cytotoxicity.

Owing to drug resistance and severe adverse drug reactions to many antibiotics, the search for new and effective antibiotic agents is essential (de León et al., 2010). Mounting evidence suggests that metabolites of endophytes can inhibit or kill a wide variety of harmful microorganisms, including phytopathogens, bacteria, fungi, viruses, and protozoans (Strobel et al., 2004). In this study, we observed the antibacterial activity of endophytes against some strains of $S$. aureus including MSSA and MRSA. We found that T-27, belonging to Xylaria sp, was the most effective against $S$. aureus strains with MICs less than 0.25 and $4 \mathrm{mg} / \mathrm{mL}$ (see Table 2). T-27 exhibited time-dependent inhibition against ATCC 25923 (MSSA) and ATCC 43300 (MRSA) with EE concentrations of $31.25 \mu \mathrm{g} / \mathrm{mL}$ and $1 \mathrm{mg} / \mathrm{mL}$, respectively, according to the bacterial dynamic growth curves shown in Figures 1 and 2. Recently, an endophytic $X y$ laria sp isolated from $G$. biloba L. has been shown to have broad antimicrobial activity (Liu et al., 2008); its metabolites contained the antimicrobial compound 7-amino-4-methylcoumarin. Furthermore, crude EEs of Xylaria sp strains (Ascomycetes) showed dramatic antibacterial activity against MRSA strains (Ramesh et al., 2012), suggesting that T-27 consumes and transfers the composition of the culture medium to produce antibacterial metabolites.

In conclusion, our results demonstrated that the endophytes in $S$. verrucosa exhibited moderate antibacterial activity and potent cytotoxicity, suggesting that they are a source for the production of natural bioactive products and thus warrant further investigation. 


\section{ACKNOWLEDGMENTS}

Research was conducted in the Class III Laboratory of Traditional Chinese Medicine on Pharmacognosy of State Administration of Traditional Chinese Medicine of the People's Republic of China, Academy of Integrative Medicine, Fujian University of Traditional Chinese Medicine, Fuzhou, Fujian, China. Research supported by the Key Project of Fujian Provincial Universities for Haixi Development (Grant \#5), and the Research Foundation of Education Bureau of Fujian Province of China (\#JA10182). We are grateful to Dr. Lu Ping Qin (Second Military Medical University, Shanghai, China) for assistance in identifying endophytic.

\section{REFERENCES}

Aly AH, Debbab A, Clements C, Edrada-Ebel R, et al. (2011). NF kappa B inhibitors and antitrypanosomal metabolites from endophytic fungus Penicillium sp. isolated from Limonium tubiflorum. Bioorg. Med. Chem. 19: 414-421.

de León L, Lopez MR and Moujir L (2010). Antibacterial properties of zeylasterone, a triterpenoid isolated from Maytenus blepharodes, against Staphylococcus aureus. Microbiol. Res. 165: 617-626.

Gao Q and Cao T (2000). Bryophyta: Hepaticae, Anthocerotae. In: Flora Yunnanica. (Wu Zhengyi, ed.). Vol. 17. Science Press, Beijing, 247.

Ghisalberti EL (1993). Detection and Isolation of Bioactive Natural Products. In: Bioactive Natural Products: Detection, Isolation and Structure Elucidation (Colegate SM and Molyneux RJ, eds.). CRC Press, New York, 15-18.

Guo L, Wu JZ, Han T, Cao T, et al. (2008). Chemical composition, antifungal and antitumor properties of ether extracts of Scapania verrucosa Heeg. and its endophytic fungus Chaetomium fusiforme. Molecules 13: 2114-2125.

Guo L, Wu JZ, Xu QZ, Han T, et al. (2009). Screening of active strains in endophytic fungi from Scapania verrucosa using biological model of Pyricularia oryzae. Chin. Trad. Herbal. Drugs. 40: 1090-1094.

Jiang W, Li B, Zheng X, Liu X, et al. (2011). Artesunate in combination with oxacillin protect sepsis model mice challenged with lethal live methicillin-resistant Staphylococcus aureus (MRSA) via its inhibition on proinflammatory cytokines release and enhancement on antibacterial activity of oxacillin. Int. Immunopharmacol. 11: 1065-1073.

Liu X, Dong M, Chen X, Jiang M, et al. (2008). Antimicrobial activity of an endophytic Xylaria sp.YX-28 and identification of its antimicrobial compound 7-amino-4-methylcoumarin. Appl. Microbiol. Biotechnol. 78: 241-247.

McLaughlin JL, Rogers LL and Anderson JE (1998). The use of biological assays to evaluate botanicals. Drug Inform. J. 32: 513-524.

Peng W, Han T, Xin WB, Zhang XG, et al. (2011). Comparative research of chemical constituents and bioactivities between petroleum ether extracts of the aerial part and the rhizome of Atractylodes macrocephala. Med. Chem. Res. 20: 146-151.

Qin JC, Zhang YM, Gao JM, Bai MS, et al. (2009). Bioactive metabolites produced by Chaetomium globosum, an endophytic fungus isolated from Ginkgo biloba. Bioorg. Med. Chem. Lett. 19: 1572-1574.

Ramesh V, Arivudainambi U, Thalavaipandian A, Karunakaran C, et al. (2012). Antibacterial activity of wild Xylaria sp. strain R005 (Ascomycetes) against multidrug-resistant Staphylococcus aureus and Pseudomonas aeruginosa. Int. J. Med. Mushrooms. 14: 47-53.

Solis PN, Wright CW, Anderson MM, Gupta MP, et al. (1993). A microwell cytotoxicity assay using Artemia salina (brine shrimp). Planta Med. 59: 250-252.

Söderström L, Séneca A and Santos M (2007). Rarity patterns in members of the Lophoziaceae/Scapaniaceae complex occurring north of the tropics - implications for conservation. Biol. Conserv. 135: 352-359.

Stierle A, Strobel G and Stierle D (1993). Taxol and taxane production by Taxomyces andreanae, an endophytic fungus of Pacific yew. Science 260: 214-216.

Strobel G, Daisy B, Castillo U and Harper J (2004). Natural products from endophytic microorganisms. J. Nat. Prod. 67: 257-268.

Strobel GA (2002). Rainforest endophytes and bioactive products. Crit. Rev. Biotechnol. 22: 315-333.

Tan RX and Zou WX (2001). Endophytes: a rich source of functional metabolites. Nat. Prod. Rep. 18: 448-459.

Wang QX, Li SF, Zhao F, Dai HQ, et al. (2011). Chemical constituents from endophytic fungus Fusarium oxysporum. Fitoterapia 82: 777-781.

Weber RW, Kappe R, Paululat T, Mosker E, et al. (2007). Anti-Candida metabolites from endophytic fungi. Phytochemistry 
68: 886-892.

Xu LL, Han T, Wu JZ, Zhang QY, et al. (2009). Comparative research of chemical constituents, antifungal and antitumor properties of ether extracts of Panax ginseng and its endophytic fungus. Phytomedicine 16: 609-616.

Yin H and Sun YH (2011). Vincamine-producing endophytic fungus isolated from Vinca minor. Phytomedicine 18: 802805.

Zeng PY, Wu JG, Liao LM, Chen TQ, et al. (2011). In vitro antioxidant activities of endophytic fungi isolated from the liverwort Scapania verrucosa. Genet. Mol. Res. 10: 3169-3179. 